\title{
Primer Seminario sobre agricultura y alimentación (Conclusiones)
}

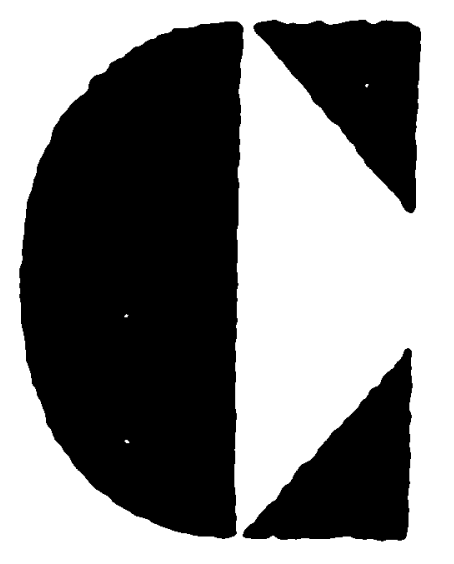

ON EL auspicio de la Fundación Friedrich Ebert $y$ de la Fundación para el Desarrollo Nacional

Proyecto SINEA, el Programa Académico de Ciencias Sociales de la Pontificia Universidad Católica del Perú organizó el Primer Seminario sobre Agricultura y Alimentación.

Durante tres dias (del 10 al 13 de octubre) más de cien especialistas escogidos por su versación $y / 0$ experiencia práctica en el tema, procedentes de organismos públicos, universidades, centros de investigación $e$ instituciones internacionales, trabajando concentrados en Chaclacayo 10 horas cada jornada, escucharon quince ponencias, las discutieron $y$ comentaron. El debate mostró más punto de convergencia de los que originalmente pudo pensarse dada la diversidad de especialidades - agrónomos, ecólogos, nutricionistas, medicos, abogados, antropólogos, economistas, sociólogos, etc.- $y$ el natural abanico político implicado. (Se invitó además a representantes de organismos de productores $y$ dirigentes políticos).

Una Comisión de Redacción y el Comité Organizador del Seminario han preparado $y$ aprobado estas primeras conclusiones que resumen los puntos básicos de convergencia antes mencionados. Ellos. comprenden una apreciación de la situación y ciertos lineamientos básicos de una politica agroalimentaria alternativa. Posteriormente, un libro recogerá el valioso material de ponencias e intervenciones de panel que dieron contenido al Seminario. El comprenderá: una segunda y más completa entrega de conclusiones.

En conjunto la reunión ha constituido un primer paso que tratará de ser continuado. El Programa de Ciencias Sociales de la Universidad Católica agradece la respuesta generosa y calificada de todos los participantes y ratifica su voluntad de aportar espacios de discusión y proporciones que resultan del trabajo científico nacional sobre los distintos $y$ muchas veces dramáticos aspectos de nuestra realidad.

Lima, 18 de octubre, 1979 


\section{CONCLUSIONES}

(Primera Entrega)

\section{LA SITUACION ALIMENTARIA}

Todos los datos disponibles indican que un porcentaje superior al 500/o de la población del Perú tiene un consumo deficidario de alimentos, y que esta situación se ha agravado en los últimos años.

Un análisis de las caracteristicas de este gravísimo proceso conduce a cuestionar el patrón de crecimiento económico-y agrario en particularasí como las respectivas politicas que se han implementado en los últimos decenios.

Lus sectores sociales que hoy presentan una situación nutricional más dramática son los estratos más pobres de las ciudades, principalmente Lima. Esto ocurre pese a que las politicas económicas han tratado de favorecer a la ciudad frente al campo.

Estas constataciones contradicen la creencia de que el crecimiento y modernización de la economía traen consigo una clevación automática de los niveles de vida. Cuando ese crecimiento ocurre en el marco de un sistema que no está orientado al bienestar de toda la población, da como resultado, empobrecimiento. desnutrición y miseria para amplios sectores ciel pais.

En este sentido el Seminario puso en evidencia que el problema de la alimentación no puede reducirse sólo a ser aspectos de nutrición, producción de alimentos $e$ ingresos monetarios, sino que depende fundamentalmente del modelo integral de desarrollo de la sociedad.

\section{Mercado y Abastecimiento}

En la base del problema alimentario actual está el mecanismo del mercado y su escala internacional. Es asi que consumimos principalmente productos alimenticios que son ofertados por las empresas multinacionales, que dominan el sistema mundial de alimentos. Más aún, esta situación que beneficia ante todo a tales empresas, es apoyada por la política económica del Estado a través de medidas cambiarias, crediticias y de subsidios directos.

La tecnología de estas empresas se ha especializado en cereales, productos para los que el país no tiene demasiadas condiciones. La presencia masiva de estos alimentos, en especial en las ciudades, ha contribuido a fomentar ciertos hábitos de consumo que tienden a perpetuar tal situación, $y$ desalientan el desarrollo de la agricultura nacional. Se ha venido conformando así, una dieta promedio con alto contenido de materias primas importadas.

El hecho de reducir la politica alimentaria a una politica de abastecimiento urbano, dejando de 
lado el foménto de la agricultura nacional, lleva en el largo plazo no sólo al deterioro de esta producción $y$ de los ingresos reales de los campesinos sino también a acrecentar el problema del abastecimiento mismo, al quedar éste enteramente dependiente de la fluctuación de los precios en el mercado mundial.

Pero no se trata únicamente de. casos de este tipo. Una situación particular dramática la constituye el abandono de la lactancia materna debido a campañas comerciales interesadas, y a su reemplazo por productos industrializados de calidad inferior $y$ con efectos negativos en la alimentación y en la salud infantil.

\section{ECOLOGIA, REGIONES Y FORMAS DE PRODUCCION}

La base ecológica que ofrece el territorio peruano para la produc. ción de alimentos es una de las más variadas del mundo, pero presenta enormes limitaciones $y$-dificultades para su utilización. Esta restricción exigió esfuerzos gigantescos de los peruanos durante siglos, para crear y aprovechar los recursos necesarios para la producción alimentaria.

Con enorme ingenio y tesón desarrollaron un amplio repertorio de tecnologias agrarias yue permitieron la ocupación productiva de los diferentes espacios ecológicos del territorio nacional. Esas tecnologias constituyen un preciado legado cultural y una base técnica recuperable en muchos aspectos dentro de un proceso de desarrollo agrario auténticamente nacional.

Sin embargo, en los últimos decenios, muchos de esos recursos, al ser explotados bajo modalidades dirigidas al lucro privado, sufren rápidamente una grave degradación, muchas veces irreversible.

\section{Costa}

En la Costa peruana la restricción fundamental es el agua. De una descarga anual de 40,000 millones de $\mathrm{m} 3$ se aprovechan actualmente 10,000 millones, cifra yule con las actuales técnicas podria aumentarse sólo en un 600/o. La concentración de la descarga en un periodo muy breve cada año, impide un mejor aprovecinamiento del agua vajo dicilas fórmulas.

En esta vía de iricorporación de nuevas tierras que se traduce en costosos trabajos de irrigación, además de los problemas de financiamiento, muchas veces se añaden también problemas de drenaje y salinización progresiva. A pesar de ello el Presupuesto del sector agrario está dedicado en más de un 800/0 a este tipo de obras, en las que, además, tanto por los términos habituales de los préstamos como por la política del Estado, se lescuida por completo su mantenimiento y conservación. 
Sin embargo, hay alternativas factibles, como es el caso de las técnicas de riego longitudinales en las que en lugar de almacenar el agua se procede a su clispersión controlada, habilitando tierras para cierto tipo de cultivos. Obviamente esto requeriría no sólo una tecnología menos costosa y sofisticada, sino también otras formas de organización de la producción y de tenencia de la tierra.

Sierra

En cuanto a la actividad agropecuaria en la Sierra, si bien tiene dificultades para expandirse, es factible desplegar un esfuerzo centrado principalmente en el desarrollo cualitativo y cuantitativo de la agricultura de secano. Puede así convertirse para la agricultura amplias extensiones actualmente utilizadas en pastoreo extensivo.

\section{Selva}

La afirmación habitual de que la Selva y la Ceja de Selva ofrecen ilimitadas posibilidades agropecuarias no corresponde a la realidad. En primer lugar, por la muy baja productividad económica de los bosques tropicales húmedos, debida a su vez a la falta de una tecnología adecuada para su uso integral. En segundo lugar, porque la explotación realizada en las últimas décadas, sobre todo en la
Ceja de Selva ha degradado irreversiblemente enormes extensiones de tierras (alrededor de 4 millones de hectáreas según los estudios actualmente disponibles). Quedan sin embargo en la región de la Selva Alta y Baja, alrededor de 3 millones de hectáreas aptas para la agricultura, cuyo aprovechamiento racional permitiría duplicar la superficie agrícola actual del país, siempre que no prosiga su depredación.

\section{Una actitud Colonialista}

El tratamiento de los recursos naturales por las empresas extranjeras, por muchos productores peruanos y aún por el Gobierno, ha evidenciado una mentalidad calificable de colonialista,, interesada en extraer el máximo beneficio inmediato sin preservar el sistema ecológico global.

\section{EL ESTADO Y LOS PRODUCTORES}

\section{Política Agraria}

La política agraria seguida en los últimos tiempos ha utilizado instrumentos diversos con los que ha influido sobre la comercialización, y. los precios, por ejemplo a través de impuestos y subsidios. Se constate en general que ha contribuído a un desbalance en la producción en beneficio de los 
sectores urbanos de mayores ingresos, y en desmedro del campesinado $y$ de la población de escasos recursos en general. Es necesario enfatizar la falta de una politica integral sobre la alimentación popular.

\section{Estructura del Estado}

El comportamiento del Estado con respecto al agro $y$ a la producción de alimentos ha estado trabado por una innecesaria $y$ desordenada complejidad administrativa, además de una actitud reglamentaria, tanto sectorial como intersectorial, que ha dificultado la formulación $y$ aplicación de políticas coherentes. Se desprende de esto que en lo sucesivo el Estado deberá asumir una organización mucho más simplificada, centrándose menos en los aspectos puramente controlistas, $y$ dando más atención a la promoción y a la planificación de la producción.

Los Productores Directos y sus Organizaciones

Esta estructura y comportamiento del aparato estatal ha funcionado prescindiendo de los productos directos y sus organizaciones. Esta ausencia ha sido un elemento importante de la politica agraria misma llevada adelante por el Estado. Las organizaciones que han intervenido a raiz del proceso de Reforma Agraria han estado sujețas a una creación desde arriba, la cual ha demostrado no ser una vía adecuada. Se desprende también de esto último que una simplificación y agilización del aparato estatal deberá lograrse en correspondencia con una presencia activa de las organizaciones de los productores directos, libremente generadas, en la gestión agrícola en su conjunto.

Cabe destacar principalmente el papel que la comunidad campesina debe llegar a tener en la reconstrucción de las estructuras agrarias, así como en todo el proceso conducente a esta meta, incluyendo necesariamente las actividades de investigación $y$ extensión.

\section{EL CONOCIMIENTO DE LA REA- LIDAD AGRARIA Y SU APLI- CACION}

\section{Producción y Tecnología}

Los estudios y experiencias disponibles, indican la existencia de un potencial de posibilidades productivas actualmente desaprovechadas por circunstancias diversas, entre las que se pueden mencionar:

- La imposición indiscriminada de tecnologías que se aplican en distintas ocasiones fuera de las circunstancias en las que actuarían eficientemente. 
- Abandono de técnicas y recursos nacionales en sanidad vegetal, fertilizantes y otros campos, ante la difusión indiscriminada de sustitutos del mercado internacional cuyos efectos traen graves consecuencias para el equilibrio vital de los ecosistemas.

- Presencia de una ideología que lleva a admitir, sin mayor examen crítico, tecnologias que se presumen universales. Por lo mismo, se constata la necesidad de mocificar en este aspecto la orientación de la formación profesional actualmente predominante, en campos tales como nutrición, medicina, agronomía, zootecnia, ingeniería agrícola, agrometereologia, etc.

\section{Información e Investigación}

El desarrollo del Seminario ha permitido precisar caracteristicas ciel sector ayrario nacional y de su evolución en las últimas décadas que contrastan en importantes aspectos con la imagen que ha manejado el Estado durante los últimos años incluido el periodo de la Reforma Agraria. Es indudable que esta circunstancia perjudica la formulación e implementación de políticas, al no corresponder la imagen a la realidad. El esfuerzo intelectual y técnico de los últimos anos ha permitido un mejor conocimiento de la problemática agraria y ha destacado por ejemplo la articulación entre el estancamiento de la agricultura y el modelo de industrialización y de desarrollo global predominante en el pais, articulación aún hoy no suficientemente comprendida.

Cabe señalar además que las fuentes de información disponibles han permanecido y en muchos casos siguen permaneciendo fuera del alcance de las instituciones y personas dedicadas al estudio e investigación de los distintos aspectos de nuestra realidad, sin que en la gran mayoria de los casos exista razón alguna para este ocultamiento. Muy por el contrario, se impone un apoyo decidido a la labor de investigación y extensión.

\section{CONCLUSION: NECESIDAD DE UN PROYECTO NACIONAL}

Es factible, en realidad, desarrollar la producción ágropecuaria .en las tres regiones, especialmente en Sierra y Selva, dentro de patrones tecnológicos y de organización de la producción adecuados, que impidan la continuación dé la de. predación de los recursos.

Perc) esta posibilidad requicre como requisito indispensable el que los recursos económicos sean explotalos s desarrollados en ráón de un proyecto genuinamente nacional, destinado al bienestar de la población peruana en su conjunto.

Tal proyecto debe asignar como 
objetivo central de la producción agraria nacional, la provisión creciente de los alimentos que el país requiere. El logro de este objetivo exige dar prioridad en la política agraria al apoyo a la agricultura campesina, especialmente en la Sierra. En la actualidad, pese a todas las dificultades, que han conducido al deterioro de su producción, los pequeños productores camipesinos son los que proporcionan la mayor parte de los alimentos del país. Políticas apropiadas al respecto podrian tener un efecto beneficioso sobre esta producción. Para ello se requiere adecuar el uso de tecnologias tanto tradicionales como modernas según las caracteristicas de los diferentes medios ecológicos y sociales, así como promover las formas de organización y decisión que aseguren el desarrollo de la producción campesina.
En el Seminario se reconocieron las implicaciones políticas indisociables de esta búsqueda de un nuevo curso en la organización de la producción y el consumo agroalimen-, tario.

Estas conclusiones ponen énfasis en las consideraciones de conjunto que constituyeron el principal tema del Seminario. Se hace público un cuestionamiento de la actual tendencia en la producción, la políticá alimentaria y el consumo y se afirma la viabilidad y urgencia de una dirección global alternativa. Algunos lineamientos fundamentales para esta alternativa son los que quedan sintetizados en el presente documento. La publicación posterior del conjunto del trabajo del Seminario contendrá recomendaciones más puntuales y una más amplia fundamentación de las tesis que aqui hemos resumido. 